\title{
An efficient chronic unpredictable stress protocol to induce stress-related responses in C57BL/6 mice
}

\section{Susana Monteiro ${ }^{1,2}$, Susana Roque ${ }^{1,2}$, Daniela de Sá-Calçada ${ }^{1,2}$, Nuno Sousa ${ }^{1,2}$, Margarida Correia-Neves ${ }^{1,2}$ and João José Cerqueira ${ }^{1,2}$ *}

1 Life and Health Sciences Research Institute (ICVS), School of Health Sciences, University of Minho, Braga, Portugal

2 ICVS/3B's Research Group - PT Government Associate Laboratory, Braga, Portugal

\section{Edited by:}

Stefan Oskar Reber, University of

UIm, Germany

\section{Reviewed by:}

Catherine Belzung, Université

Francois Rabelais, France

Mathias V. Schmidt, Max Planck

Institute of Psychiatry, Germany

James P. Herman, University of

Cincinnati, USA

\section{*Correspondence:}

João José Cerqueira, ICVS/3B's

Research Group - PT Government

Associate Laboratory, Life and Health

Sciences Research Institute (ICVS),

School of Health Sciences, University

of Minho, Campus de Gualtar, Braga

4710-057, Portugal

e-mail: jcerqueira@ecsaude.

uminho.pt
Exposure to chronic stress can have broad effects on health ranging from increased predisposition for neuropsychiatric disorders to deregulation of immune responses. The chronic unpredictable stress (CUS) protocol has been widely used to study the impact of stress exposure in several animal models and consists in the random, intermittent, and unpredictable exposure to a variety of stressors during several weeks. CUS has consistently been shown to induce behavioral and immunological alterations typical of the chronic stressresponse. Unfortunately C57BL/6 mice, one of the most widely used mouse strains, due to the great variety of genetically modified lines, seem to be resistant to the commonly used 4-week-long CUS protocol. The definition of an alternative CUS protocol allowing the use of C57BL/6 mice in chronic stress experiments is a need. Here, we show that by extending the CUS protocol to 8 weeks is possible to induce a chronic stress-response in $\mathrm{C} 57 \mathrm{BL} / 6$ mice, as revealed by abrogated body weight gain, increased adrenals weight, and an overactive hypothalamic-pituitary-adrenal axis with increased levels of serum corticosterone. Moreover, we also observed stress-associated behavioral alterations, including the potentiation of anxious-like and depressive-like behaviors and a reduction of exploratory behavior, as well as subtle stress-related changes in the cell population of the thymus and of the spleen. The present protocol for C57BL/6 mice consistently triggers the spectrum of CUS-induced changes observed in rats and, thus, will be highly useful to researchers that need to use this particular mouse strain as an animal model of neuropsychiatric disorders and/or immune deregulation related to CUS.

Keywords: chronic stress, CUS, neuropsychiatric disorders, immune dysfunction, anxiety, depressive-like behavior, social defeat

\section{INTRODUCTION}

Stressful life events can be triggering factors of numerous neuropsychiatric disorders namely anxiety, depression, and dementia (1), and many of these are accompanied by immune dysfunction (2). Moreover, prolonged-stress-induced immune dysfunction itself is regarded as a contributing factor for the effects of stress on health (3). In contrast with chronic stress, the acute stress-response is a beneficial event since it is an alarm reaction that prepares the body to a possible threat. This response is characterized by the secretion of stress mediators, such as glucocorticoids and epinephrine, which allows the stability of body function by adaptation to the stressor (4). However, when this response persists in time, it might render the system unable to cope with the stressor, ultimately leading to chronic-stress-associated illness.

Neuropsychiatric alterations are the most widely described effects of chronic stress exposure and include anxious-like behavior (5-8), depressive-like behavior $(9,10)$, and cognitive deficits $(5,11-14)$. However, the effects of chronic stress are not only limited to behavioral changes. Immune cells express receptors for glucocorticoids and catecholamines $(15,16)$, which can lead to alterations in gene transcription in response to stress (17). In fact, it is generally accepted that chronic-stress-associated changes in the immune system alter the vulnerability to infectious disease and auto-immunity (18).

Stress exposure variables, such as duration, intensity, and predictability, explain the spectrum of differential responses to stress but ultimately, the threshold in which the stressresponse switches from physiological to deleterious is also dependent on neuroendocrine, neurochemical, and genetic factors that are responsible for individual differences in stress perception and response (19). Having this in mind, it seems logical that for the use of animal models, the chronic stress protocol needs to be adjusted to the animal species and even the strain used.

The most commonly used unpredictable chronic stress paradigms are the unpredictable chronic mild stress (uCMS) and the chronic unpredictable stress (CUS). Although both terms, uCMS and CUS, tend to be used indiscriminately nowadays and that both protocols are widely used to study depression, the original purpose for which they were generated was quite distinct. uCMS paradigm have been long used to model depression, and consists in the continuous exposure of animals to stressful situations, usually for at least 4 weeks, including some stressors that 
involve water and/or food deprivation. In contrast, CUS was originally used to study mechanisms underlying the stress-response and involves the intermittent exposure to a daily stressful stimulus, lasting at least 4 weeks, being one of the main advantages of this protocol the absence of stressors that interfere with water and/or food deprivation, which might better mimic everyday life stress.

Although rats are widely used as animal models of depression and other stress-related disorders, mice present advantages such as the availability of numerous genetically modified strains like transgenic and KO mice and the lower maintenance costs when compared to rats. Unfortunately, the most widely used inbreed strain of genetically modified mice, the C57BL/6, seems to be less vulnerable to stress than other mouse strains (20-25).

Our aim was to develop an improved CUS protocol to be used in C57BL/6 mice. In order to do so we modified the standard CUS protocol by including social defeat stress as one of the stressors and extending its duration to 8 weeks. By comparing the neuroendocrine, behavioral, and immune changes induced by the unmodified 4-week long CUS exposure and the optimized 8-week long CUS protocol we, herein, show the advantages of later for C57BL/6 mice.

\section{MATERIALS AND METHODS \\ ANIMALS}

Male C57BL/6 mice (C57BL/6J JAX ${ }^{\mathrm{TM}}$ mice strain) were purchased from Charles River (Charles River Laboratories, Barcelona, Spain) and housed (five animals per cage) under standard laboratory conditions ( $12 \mathrm{~h}$ light $/ 12 \mathrm{~h}$ night cycles $(8 \mathrm{~h} / 20 \mathrm{~h}), 22-24^{\circ} \mathrm{C}$, relative humidity of $55 \%$ and ad libitum access to water and food. All procedures were carried out in accordance to EU directive 2010/63/EU and Portuguese national authority for animal experimentation, Direção Geral de Veterinária (ID:DGV9457) guidelines on animal care and experimentation.

\section{CHRONIC UNPREDICTABLE STRESS PARADIGM}

One group of C57BL/6 animals was exposed to 4 weeks of CUS and compared to a control group that was subjected to gentle handling, twice a week, for the same period. Another group was exposed to 8 weeks of CUS and compared to other control group that was subjected to gentle handling, twice a week, for the same period. Mice were 8-week old when the CUS protocol was initiated. Each group consisted of 10-15 male C57BL/6 mice. We run two independent experiments to confirm our findings: data from the first, representative of our findings, are presented in the main paper, whereas data from the second experiment are shown as supplementary data (Figure S1 and Table S1 in Supplementary Material).

Briefly, the CUS paradigm consisted in exposure, once daily, to one of the following aversive stressors: restraint - mice were placed in a $50 \mathrm{ml}$ plastic tube (Falcon) with openings in both sides for breathing, for $1 \mathrm{~h}$; shaking - groups of five mice were placed in a plastic box container and placed in an orbital shaker for $1 \mathrm{~h}$ at $150 \mathrm{rpm}$; social defeat - mice were introduced in a cage of an aggressive mice and after being defeated, they were placed in a transparent and perforated plastic container, to avoid further physical contact, inside the resident homecage for $30 \mathrm{~min}$ (26); hot air stream - mice were exposed to a hot air stream from a hairdryer for $10 \mathrm{~min}$; overnight illumination - mice were exposed to regular room light during the night period; inverted light cycle - regular room light was off during daytime and on during nighttime for 2 days; tilted cage - homecages were tilted in a $45^{\circ}$ angle during $1 \mathrm{~h}$. Stressors were presented in a random order in an unpredictable fashion (see Table 1). The stressors distribution for the group submitted to 4 weeks of CUS is a truncated version of Table $\mathbf{1}$. Body weight was monitored once a week and post-mortem thymus and adrenal weight were recorded.

\section{CORTICOSTERONE QUANTIFICATION}

Blood was collected through the tail by venopuncture within a maximum $120 \mathrm{~s}$ period since removal of each mouse from its homecage to the end of blood collection. Sera were separated by centrifugation at $13000 \mathrm{rpm}$, during $5 \mathrm{~min}$ and stored at $-80^{\circ} \mathrm{C}$. Serum corticosterone levels were measured on sera collected at nadir phase (9:00 a.m.) and at zenith phase (8:00 p.m.) using a commercial radioactive immunoassay kit (MP Biomedicals, CA, USA).

\section{BEHAVIORAL ASSESSMENT}

Mice were transported and left for habituation to the testing room for $1 \mathrm{~h}$ prior to the behavioral test. The order of the behavioral tests was: elevated-plus maze (EPM) and open field (OF) (Day 1), forced swimming test (FST) and tail-suspension test (TST) (Day 2), and Morris water maze (MWM) (Day 3-7).

\section{Elevated-plus maze}

Anxious-like behavior was assessed using the EPM test (27). Briefly, this test consists on placing each mouse in the hub of a plus-like apparatus elevated $72.4 \mathrm{~cm}$ from the floor, with two opposing open arms $(50.8 \mathrm{~cm} \times 10.2 \mathrm{~cm})$ and two opposing closed arms $(50.8 \mathrm{~cm} \times 10.2 \mathrm{~cm} \times 40.6 \mathrm{~cm})$ (ENV560; Med Associates, Inc., Vermont, USA) and letting the animal freely explore it for $5 \mathrm{~min}$. Time in the open arms and in the closed arms was used as a behavioral parameter of anxious-like behavior. EPM data from one animal from each group were not included in the analysis due to failure of the video recording system.

\section{Open field}

Locomotor and exploratory activities were assessed using the OF. Each mouse was left in the center of a squared arena $(43.2 \mathrm{~cm} \times 43.2 \mathrm{~cm})$, which the mouse was free to explore for $5 \mathrm{~min}$. This arena is equipped with infrared beams for activity detection (Med Associates, Inc., Vermont, USA). Data were collected using the activity monitor software (Med Associates, Inc., Vermont, USA). Distance traveled was used as a measure of locomotor activity and the number of vertical counts as a measure of exploratory activity.

\section{Forced swimming test and tail-suspension test}

Depressive-like behavior was assessed through the FST as described by Ref. (28) and through the TST (29). Briefly, in the 
Table 1 | Example of stressors distribution.

\begin{tabular}{|c|c|c|c|c|c|c|c|}
\hline & Mon & Tue & Wed & Thu & Fri & Sat & Sun \\
\hline Week 1 & BW hot drier & Shaking & Restraint & Social defeat & Restraint & Restraint & Tilted cage \\
\hline Week 2 & BW restraint & Shaking & Social defeat & Restraint & Shaking & Social defeat & Restraint \\
\hline Week 3 & BW restraint & Social defeat & Restraint & BC-zenith & Social defeat & Restraint & Shaking \\
\hline \multirow[t]{3}{*}{ Week 4} & BW & EPM & FST & Shaking & Restraint & Shaking & Hot drier \\
\hline & $\mathrm{BC}$ - nadir & OF & TST & & & & \\
\hline & Social defeat & Restraint & Social defeat & & & & \\
\hline Week 5 & BW restraint & $\begin{array}{l}\text { Sacrifice } 4 \text { weeks } \\
\text { tilted cage }\end{array}$ & $\begin{array}{l}\text { Cytometry hot } \\
\text { drier }\end{array}$ & Restraint & Social defeat & Inverted light & Inverted light \\
\hline Week 6 & BW hot drier & Shaking & Restraint & Inverted light & $\begin{array}{l}\text { Overnight } \\
\text { illumination }\end{array}$ & Restraint & Hot drier \\
\hline Week 7 & BW restraint & Social defeat & Restraint & BC-zenith & Shaking & Restraint & $\begin{array}{l}\text { Overnight } \\
\text { illumination }\end{array}$ \\
\hline \multirow[t]{3}{*}{ Week 8} & BWBC-nadir & EPM & FST & MWM shaking & MWM & MWM & MWM \\
\hline & & OF & TST & & restraint & restraint & shaking \\
\hline & & Restraint & Social defeat & & & & \\
\hline Week 9 & BW & Sacrifice 8 weeks & Cytometry & & & & \\
\hline
\end{tabular}

BW, body weight measurement; BC, blood collection; EPM, elevated-plus maze; OF, open field; FST, forced swimming test; TST, tail-suspension test; MWM, Morris water maze test.

FST each mouse was placed in an inescapable transparent cylindrical tank filled with water $\left( \pm 24^{\circ} \mathrm{C}\right)$, for $6 \mathrm{~min}$. In the TST, each animal was suspended by the tip of its tail for $6 \mathrm{~min}$. The activity of each mouse, in both tests, was recorded using a video camera. Latency (time to the first stop), mobility and immobility times were scored manually by an investigator blind to the experimental conditions, using Etholog 2.2 software (30), and used as a measure of behavioral despair. TST data from one animal were not included in the analysis due to failure of the video recording system.

\section{Morris water maze}

In order to assess spatial reference memory, mice were tested in a white circular pool $(170 \mathrm{~cm}$ diameter) filled with water $(24-$ $25^{\circ} \mathrm{C}$ ) placed in a dimly lit room. Spatial cues were placed in the walls around the pool (square, stripes, triangle, and a cross). The pool was divided in four imaginary quadrants and a hidden transparent platform was placed in one of the quadrants. Data were collected by a fixed camera placed in the ceiling and connected to a video-tracking system (Viewpoint, Champagne-au-Mont-d'Or, France).

Mice had to learn the position of a hidden platform over a period of 4 days. In each day, mice were placed facing the wall of the pool at different quadrants (north, west, south, and east) as a starting point for each trial. Each trial was completed whenever the mouse reached the platform or when $120 \mathrm{~s}$ elapsed. Latency to reach the platform (escape latency) was recorded for each trial during the 4 days.

In the fifth day, the platform was removed and a single trial of $60 \mathrm{~s}$ was performed (probe trial). The percentage of time that each mouse swam in each quadrant was recorded to confirm the acquisition of platform location through reference memory.

\section{FLOW CYTOMETRY}

Thymus and spleen (8-10 animals per group) were dissected and homogenized in supplemented Dulbecco's modified eagle medium (DMEM) with 10\% heat inactivated FCS, 10 mM HEPES buffer, $1 \mathrm{mM}$ sodium pyruvate, $2 \mathrm{mM}$ L-glutamine, $50 \mu \mathrm{g} / \mathrm{mL}$ streptomycin, and $50 \mathrm{U} / \mathrm{mL}$ penicillin (all from Invitrogen, CA, USA) in order to obtain single-cell suspensions. Splenic erythrocytes were depleted by incubating for $5 \mathrm{~min}$ with a hemolytic

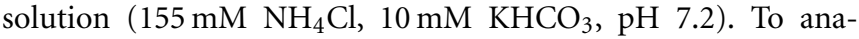
lyze the main cell populations in the thymus, the cells $\left(1 \times 10^{6}\right.$ cell) were stained with APC anti-mouse CD3 (clone 145-2C11, Biolegend, San Diego, CA, USA), V450 anti-mouse CD4 (clone RM4-5, BD Pharmingen, San Jose, CA, USA), and V500 antimouse CD8 (clone 53-6.7, BD Pharmingen, San Jose, CA, USA). Splenocytes $\left(1 \times 10^{6}\right.$ cell $)$ were stained with APC anti-mouse CD3 (clone 145-2C11, Biolegend, San Diego, CA, USA) for Tlymphocytes, PE.Cy5.5 anti-mouse CD19 (clone 6D5, Biolegend, San Diego, CA, USA) for B-lymphocytes, V450 anti-mouse CD4 (clone RM4-5, BD Pharmingen, San Jose, CA, USA) for T helper cells, V500 anti-mouse CD8 (clone 53-6.7, BD Pharmingen, San Jose, CA, USA) for T cytotoxic cells, and FITC anti-mouse NK1.1 (clone PK136, Biolegend, San Diego, CA, USA) for natural killer cells. To analyze myeloid cell populations, splenocytes $\left(1 \times 10^{6}\right.$ cell) were stained with PE anti-mouse CD11b (clone M1/70, Biolegend, San Diego, CA, USA), and PE.Cy7 anti-mouse Gr1 (clone RB6-8C5, Biolegend, San Diego, CA, USA). Cells were first gated for singlets (FSC-H vs. FSC-A) and viable cells (FSC-H vs. SSC-H). Myeloid cells were selected using the gating strategy described previously (31). Briefly, myeloid cells were gated as $\mathrm{CD}_{11} \mathrm{~b}^{+}$cells excluding the NK1.1 $1^{+}$cells. Macrophages/dendritic cells were selected as the population $\mathrm{Gr}^{+}{ }^{+} \mathrm{SSC}^{\text {low }}$, neutrophils were selected as $\mathrm{Gr}^{+}{ }^{+} \mathrm{SSC}^{\text {high }}$ and eosinophils as $\mathrm{Gr}^{-}{ }^{-} \mathrm{SSC}^{\text {high }}$ 


\section{4 weeks}

\section{A}

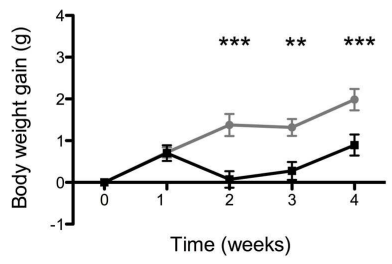

C

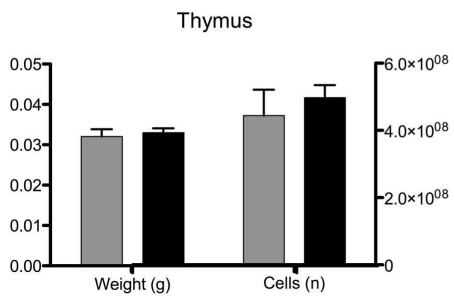

E

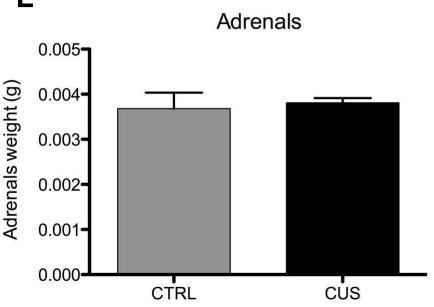

G

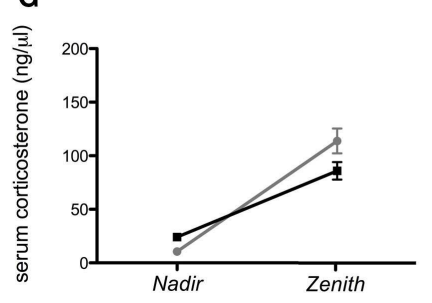

FIGURE 1 | Effect of 4 weeks vs. 8 weeks of CUS on biometric parameters. Body weight gain for animals submitted to 4 (A) and 8 (B) weeks of CUS. Thymus weight and cellularity after exposure to 4 (C) and 8 (D) weeks of CUS. Adrenals weight after 4 (E) and 8 weeks

\section{8 weeks}

B

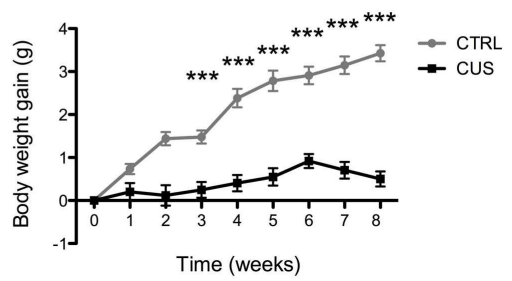

D

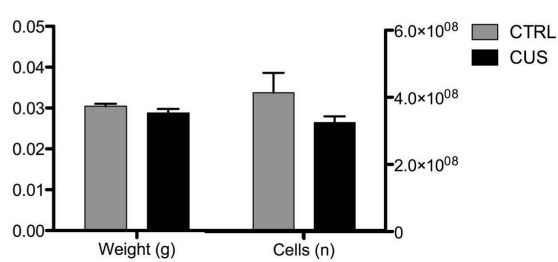

$\mathbf{F}$

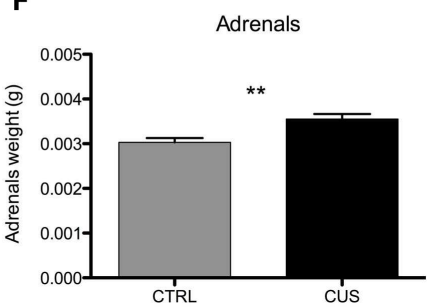

H

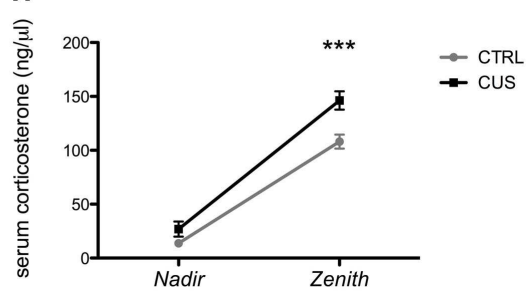

of CUS (F). Corticosterone levels in the serum of animals from the group submitted to $4(\mathbf{G})$ and $8(\mathbf{H})$ weeks of CUS. Each bar/point represents the mean \pm SEM from 10 animals per group. ${ }^{*} p<0.01$, ${ }^{* * *} p<0.001$.
(Figure 7C). After staining cells were fixed in 2\% paraformaldehyde for $20 \mathrm{~min}$. Cell surface staining was acquired $(100,000$ events) in an eight-color LSRII flow cytometer (BD, Pharmingen, San Jose, CA, USA) and analyzed with FlowJo software version 7.6.4.

\section{STATISTICAL ANALYSIS}

All values were calculated as means \pm SEM. Kolmogorov-Smirnov normality test was used to analyze if values departed from an approximate Gaussian distribution. Body weight, serum corticosterone levels, and reference memory task data were compared between groups using ANOVA repeated-measures on the average results of each week/phase/day, respectively. When the main effect was significant, post hoc Bonferroni test was performed in order to assess whether means differed significantly from each other. For all the other data, the differences among groups were analyzed using Student's $t$-test. Differences were considered significant if $p<0.05$. Statistical analysis was performed with Graphpad Prism version 5.0b (La Jolla, San Diego, USA).

\section{RESULTS}

\section{BIOMETRIC PARAMETERS AND CORTICOSTERONE MEASUREMENTS}

Body weight gain, post-mortem thymus, and post-mortem adrenal weight and serum levels of corticosterone were monitored to control for stressors efficacy (Figure 1; Figure S1 in Supplementary Material). In the group submitted to the 4-week protocol of CUS, both time $\left[F_{(4,72)}=23.85 ; p<0.0001\right]$ and exposure to CUS $\left[F_{(1,18)}=11.94 ; p=0.003\right]$ had a significant impact on body weight (Figure 1A). Moreover, there was a significant 


\section{4 weeks}
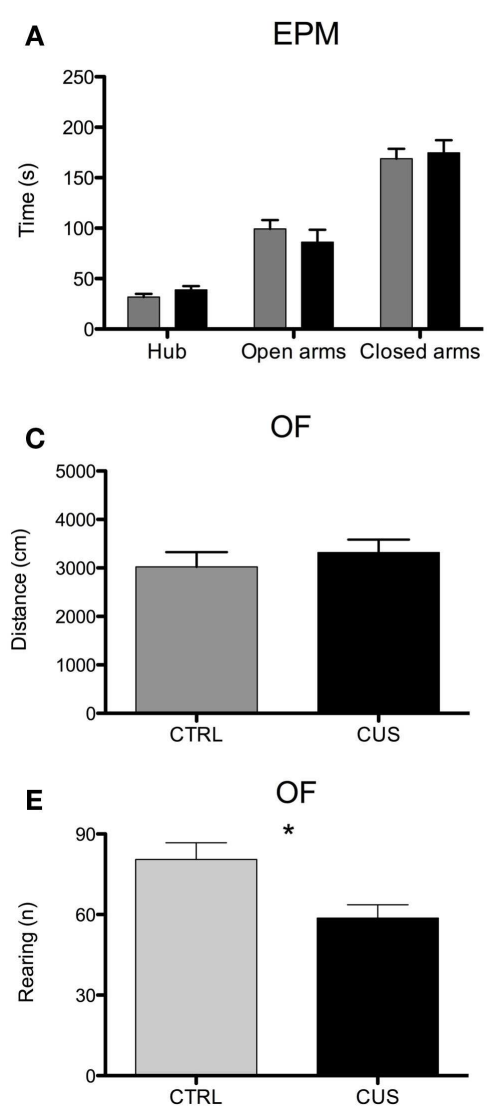

FIGURE 2 | Impact of 4 and 8 weeks of CUS on anxious-like and locomotor behavior and exploratory activity. Behavioral performance of mice exposed to 4 (A) and to 8 weeks of CUS (B) in the EPM. Locomotor function of mice submitted to 4 (C) and 8 weeks
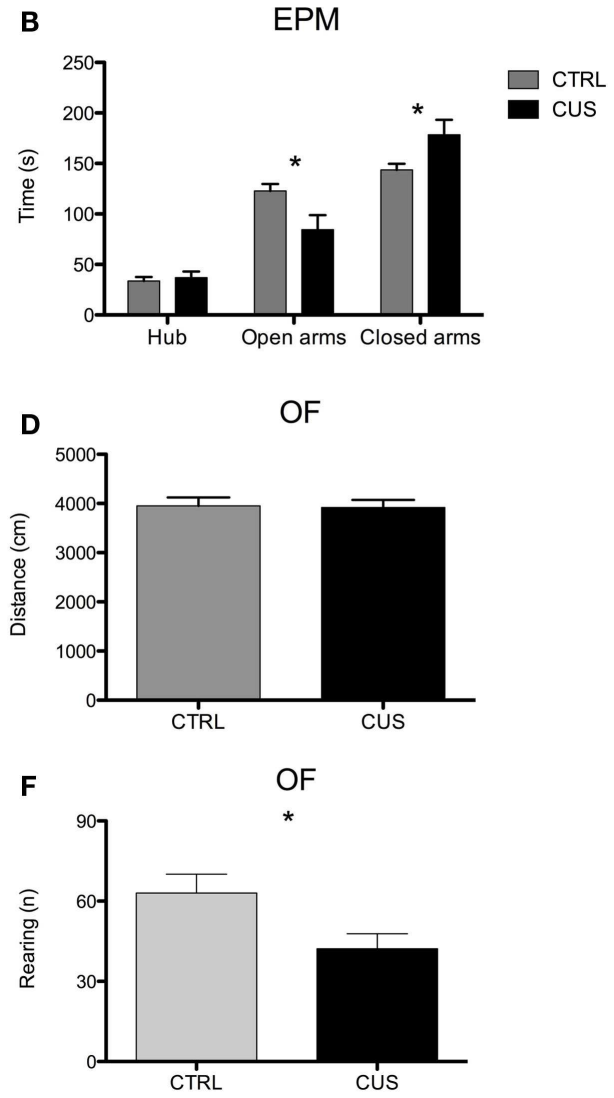

(D) of CUS measured in the OF. Exploratory activity of mice submitted to 4 (E) and 8 weeks (F) of CUS measured in the OF. Each bar represents the mean \pm SEM from 8 to 10 animals per group. ${ }^{*} p<0.05$. interaction between these factors $\left[F_{(4,72)}=23.85 ; p<0.0001\right]$ with stressed animals gaining significantly less weight over time (Figure 1A). In the group submitted to the 8-week protocol, repeated-measures ANOVA has shown again a significant effect of both time $\left[F_{(8,144)}=80.13 ; p<0.0001\right]$ and exposure to CUS $\left[F_{(1,18)}=63.43 ; p<0.0001\right]$ on body weight (Figure 1B). There was also a significant interaction between these factors $\left[F_{(8,144)}=34.17 ; p<0.0001\right]$ with stressed animals gaining significantly less weight over time (Figure 1B). CUS had no significant effect on thymus weight nor on thymic cell number, both in the group exposed to the 4- and the 8-week protocol of CUS (Figures 1C,D). CUS exposure during 4 weeks had no effect on adrenals weight, while exposure to CUS for 8 weeks led to a significant increase on adrenals weight $\left[t_{(18)}=3.449 ; p=0.003\right]$ (Figures 1E,F). There were no statistically significant changes on corticosterone levels in the group submitted to 4 weeks of CUS, both at nadir and zenith. Repeated-measures ANOVA has shown a significant effect of exposure to 8 weeks of CUS on corticosterone levels $\left[F_{(1,18)}=21.99 ; p=0.0002\right]$. Post hoc test has shown a statistically significant increase of corticosterone levels in the zenith phase of the day, in the group submitted to 8 weeks of CUS $\left[t_{(18)}=4.113 ; p<0.001\right]$ (Figures 1G,H).

\section{EXPOSURE TO 8 WEEKS OF CUS LEADS TO ALTERED EMOTIONAL BEHAVIOR BUT NOT TO MEMORY IMPAIRMENTS}

There was a significant effect of exposure to 8 but not 4 weeks of CUS on anxious-like behavior, measured by a decreased time spent on the open-arms of the EPM $\left[t_{(16)}=2.401 ; p=0.029\right]$ and an increased time spent in the closed arms $\left[t_{(16)}=2.176 ; p=0.045\right]$ (Figures 2A,B; Figures S1I,J in Supplementary Material) by the 8week CUS group when compared to controls. Exposure to CUS did not alter locomotor activity, assessed by the OF, both on the group exposed to 4 and 8 weeks of CUS (Figures 2C,D; Figures S1K,L in Supplementary Material), therefore validating behavioral tests that are dependent on an intact locomotor function. CUS had an impact on the exploratory activity, measured by a decrease on the number of rearings in the OF test, both in the group submitted to $4\left[t_{(18)}=2.743 ; p=0.013\right]$ (Figure 2E; Figure S1M in Supplementary Material) and 8 weeks of CUS $\left[t_{(18)}=2.308\right.$; $p=0.033$ ] (Figure 2F; Figure S1N in Supplementary Material). 


\section{4 weeks}

A

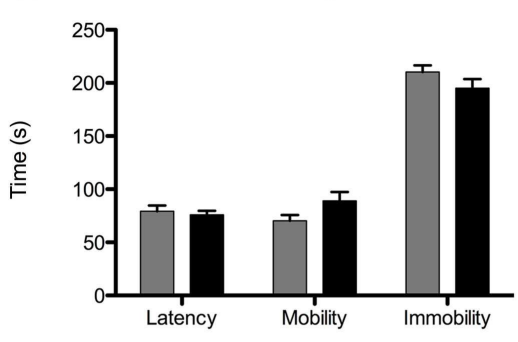

C

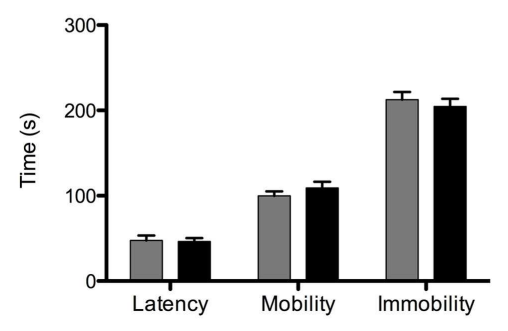

FIGURE 3 | Impact of 4 weeks vs. 8 weeks of CUS on

depressive-like behavior. Behavioral performance of mice submitted to 4 (A) and 8 weeks of CUS (B) in the FST. Behavioral performance

\section{8 weeks}

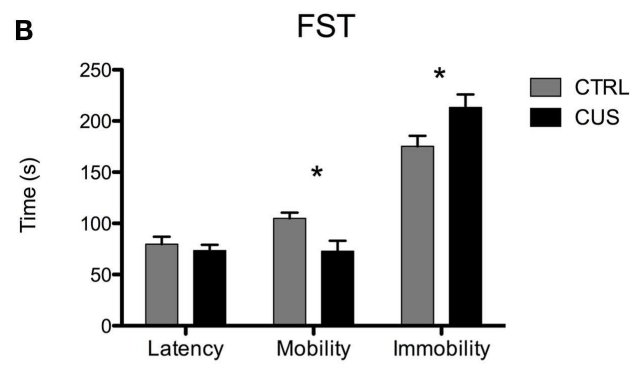

D

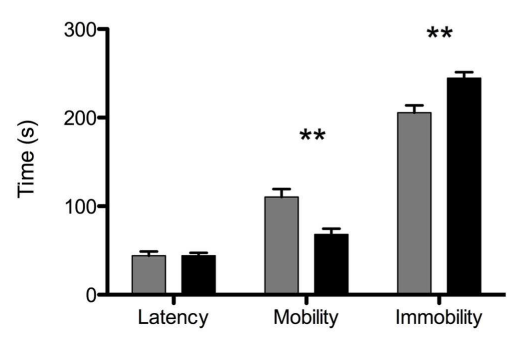

of mice submitted to 4 (C) and 8 weeks of CUS in the TST (D). Each bar represents the mean \pm SEM from 9 to 10 animals per group. ${ }^{*} p<0.05,{ }^{*} p<0.01$.
Animals exposed to 4 weeks of stress did not show any major differences in the FST when compared to controls (Figure 3A). The group of animals exposed to 8 weeks of CUS exhibited decreased mobility time $\left[t_{(18)}=2.741 ; p=0.013\right]$ and increased immobility time $\left[t_{(18)}=2.310 ; p=0.033\right]$ in the FST when compared to controls (Figure 3B). In the TST, the group submitted to 4 weeks of CUS did not show any major differences when compared to controls (Figure 3C), while the group submitted to 8 weeks of CUS exhibited a increased immobility time $\left[t_{(17)}=3.710 ; p=0.002\right]$ and an decreased mobility time $\left[t_{(17)}=3.873 ; p=0.001\right]$ (Figure 3D; Figure S1O in Supplementary Material); a typical phenotype of depressive-like behavior. No differences on latency time were found at any time point, both in the FST and TST (Figure 3).

The impact of different exposures to CUS was also tested in the MWM task in order to investigate whether the cognitive dimension was also affected. Although there was a slight tendency for a faster learning curve of the control group, especially on day 2 and 3 , in comparison to CUS exposed animals, the ANOVA repeatedmeasures test revealed that there were no significant differences between groups, meaning that, at the end of the learning task, both CUS and control groups were able to successfully learn the task therefore exhibiting an intact spatial learning ability (Figure 4; Figure S1P in Supplementary Material).

\section{THYMIC AND SPLENIC CELL POPULATION CHANGES BY EXPOSURE TO 8 WEEKS OF CUS}

It is known that thymocytes are sensitive to stress hormones, such as glucocorticoids, which modulate several processes along

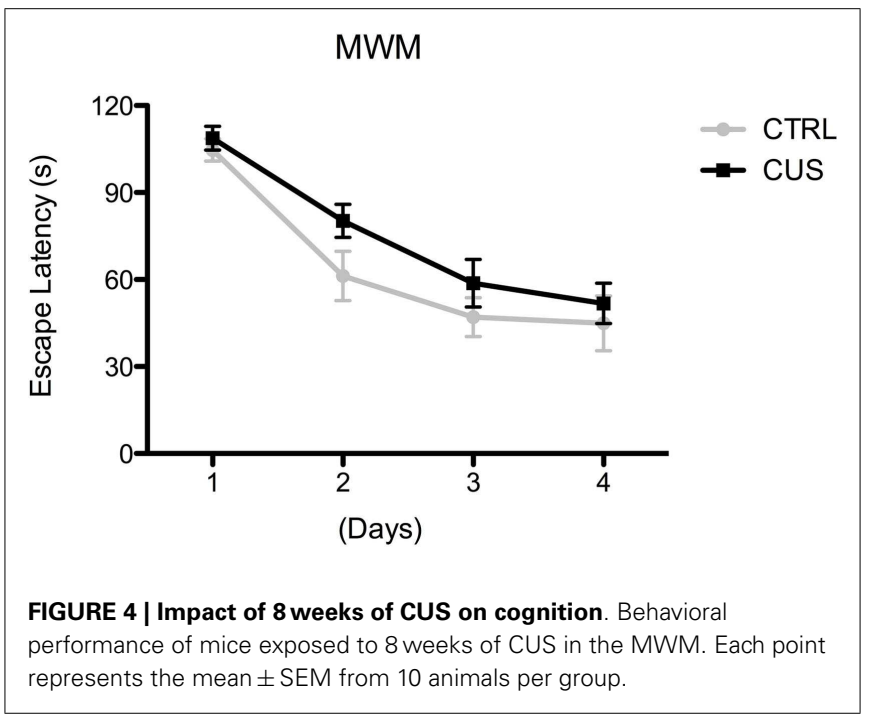

their differentiation within the thymus (32). Due to this wellknown susceptibility to stress hormones, the thymus weight, and cellularity have been widely used as indirect measures of stress. Thymocytes might be divided in four main differentiation populations depending on the expression of the CD4 and CD8 co-receptors $\left(\mathrm{CD} 4^{-} \mathrm{CD} 8^{-}\right.$double-negative $-\mathrm{DN}$; $\mathrm{CD} 4^{+} \mathrm{CD} 8^{+}$ double-positive - DP; $\mathrm{CD} 4{ }^{+} \mathrm{CD}{ }^{-}$single-positive CD4 - SPCD4; and $\mathrm{CD}^{-}{ }^{-} \mathrm{CD}{ }^{+}{ }^{+}$single-positive $\mathrm{CD} 8$ - SPCD8 cells). We therefore studied the major thymic subsets to determine if our CUS 


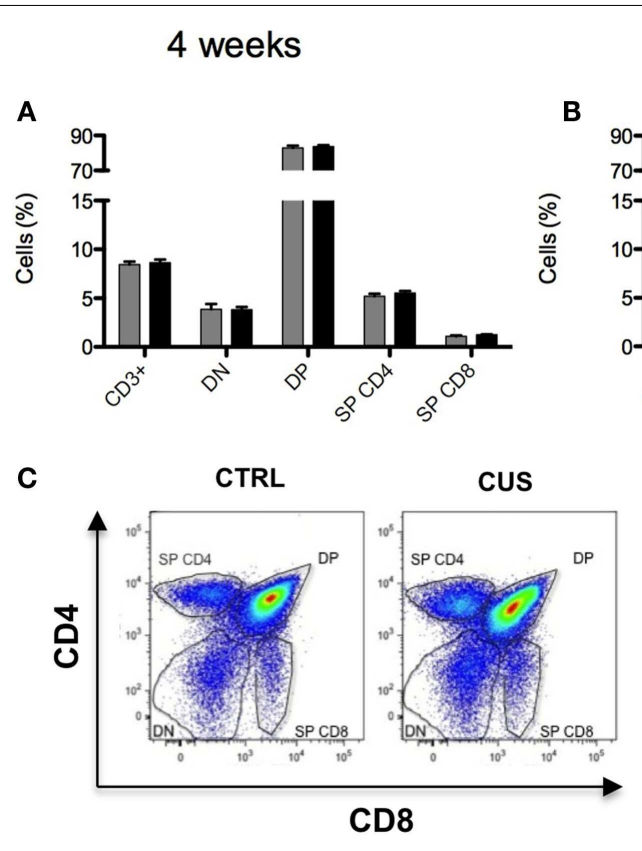

FIGURE 5 | Impact of $\mathbf{4}$ weeks vs. 8 weeks of CUS on thymocyte subsets. Percentage of main cell populations in thymus after exposure to 4 (A) and 8 (B) weeks of CUS. Flow cytometry plot showing the gating strategy for thymocyte subsets (gate) (C). DN, double-negative thymocytes; DP, double-positive thymocytes; and SPCD4 and SPCD8, single-positive CD4 and CD8 thymocytes, respectively. Each bar represents the mean \pm SEM from 10 animals per group. ${ }^{*} p<0.05$. protocols had a differential impact on them. We observed that 4 weeks of CUS did not alter the proportion of the four main thymocyte subsets (Figure 5A) while 8 weeks of stress led to an increase of the DN thymocytes proportion $\left[t_{(18)}=2.681\right.$; $p=0.020]$ (Figures 5B,C).

Since prolonged stress is known to influence the peripheral immune system we consider of relevance to investigate potential alterations caused by CUS on major lymphoid cell populations in the spleen, one of the most important lymphoid organs of the immune system. Animals exposed to 4 or 8 weeks of CUS did not show any differences on the percentage of splenic $\mathrm{T}$ and $\mathrm{B}$ cells (Figures 6A,B and E) nor in the $\mathrm{CD} 4^{+}$and $\mathrm{CD} 8^{+}$subsets among the $\mathrm{T}$ cells (Figures $6 \mathbf{C}-\mathbf{E}$ ). On the contrary, while animals exposed to 4 weeks of CUS did not show any major differences on the percentage of splenic eosinophils, neutrophils, and macrophages/dendritic cells (Figure 7A), the 8-week long CUS protocol led to an increased percentage of macrophages/dendritic cells $\left[t_{(14)}=2.188 ; p=0.046\right]$ and neutrophils $\left[t_{(14)}=3.327\right.$; $p=0.005]$ in the spleen (Figures 7B,C).

\section{DISCUSSION}

In the present work, we have optimized a CUS protocol that results in a consistent stress-response in C57BL/6 mice. Published protocols on how to induce chronic stress on rodents are diverse and generate inconsistencies in their behavioral and immunological outcomes (33). Among the main reasons for such inconsistencies are strain inherent differences of stress susceptibility/resistance to distinct protocols. In mice, specifically, the C57BL/6 strain seems to be more resistant to CUS than other strains and/or other species
(20-25). Yet, it is by far the most used mouse strain for genetic manipulations. This, and the fact that unpredictable chronic stress exposure is often used as a model of neuropsychiatric disorders, renders an effective CUS protocol in C57BL/6 mice, such as the one herein described, an important addition to the field.

Besides strain considerations, the type, diversity of stressors applied, and stress exposure length are also critical determinants of the impact of chronic stress. Some protocols use a single stressor, e.g., $6 \mathrm{~h}$ of daily restraint stress for a 4 -week period $(34,35)$, which, despite being simpler to apply, have several limitations due to lack of unpredictability or the prolonged removal of animals from their homecages with no access to food or water for half of their resting period. On the other hand, reducing restraint stress to $1 \mathrm{~h}$ per day in order to overcome this difficulty results in a mild stress protocol.

Other widely used chronic stress protocol consists in the exposure to repeated bouts of social defeat stress, which have shown to induce a stressed phenotype in some C57BL/6 mice. However, both restraint and social defeat stress paradigms are characterized by repeated exposure to a single stressor, which lacks the variability of psychological and physical stressors generally encountered in life. Taking the aforementioned into account, we designed a CUS protocol, based on the appliance of a variety of stressors, presented randomly once per day, in an intermittent and unpredictable fashion, mimicking the variability of stressors encountered on everyday life (construct validity). Although not often used in mice, CUS protocols are widely used in rats and were shown to be highly effective in inducing a stress-related phenotype $(6,11,12,36)$. In addition, by extending this protocol to 8 weeks, instead of the usual 


\section{4 weeks}

A

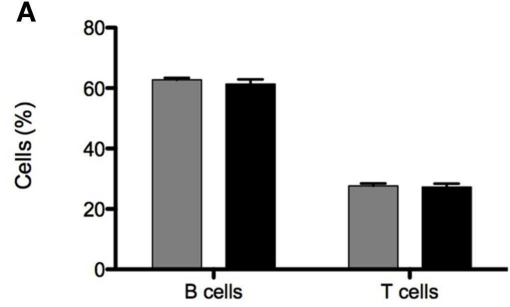

C

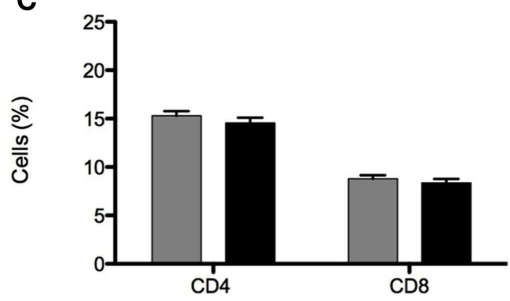

8 weeks

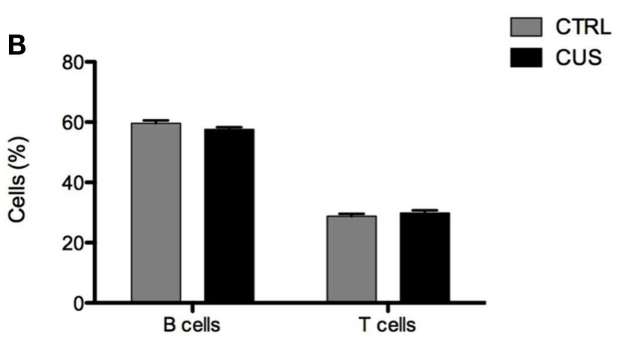

D

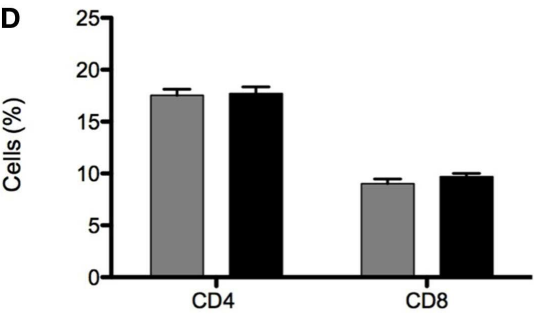

E

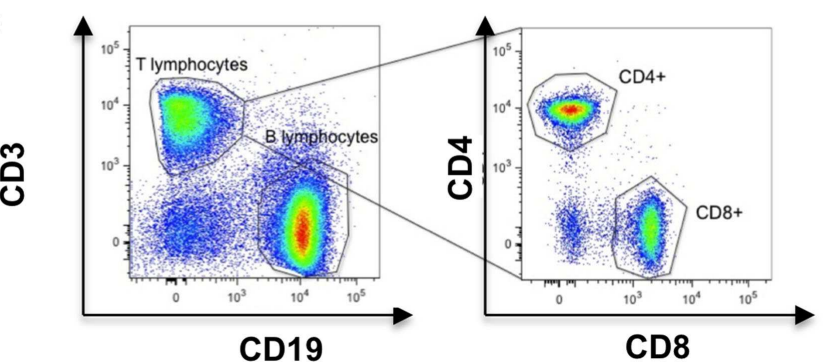

FIGURE $\mathbf{6}$ | Impact of 4 vs. 8 weeks of CUS on lymphoid cellular populations in the spleen. Percentage of $T$ and $B$ cells in spleen after exposure to $4(\mathbf{A})$ and $8(\mathbf{B})$ weeks of CUS. Percentage of $\mathrm{CD}^{+}$and $\mathrm{CD}^{+}$
T cells in spleen after exposure to 4 (C) and 8 (D) weeks of CUS. Flow cytometry plot showing the gating strategy for $\mathrm{T}$ and B-lymphocytes (gates) (E). Each bar represents the mean \pm SEM from eight animals per group.
4 weeks, we were able to reach the point where this particular strain of mice clearly and consistently exhibits a maladaptive response to chronic stress with behavioral and immunological alterations (face validity).

One of the main advantages of this 8-week long CUS protocol is that there is no stressor that implies the disturbance of food and/or water consumption, which is of particular importance for metabolism studies, for example. Moreover, in this protocol FST or TST are not used as stressors, as used in some published protocols (9), which means that in our protocol these tests can still be used as behavioral measures.

Reduction on body weight gain, thymic involution (37-39), and increased adrenals weight (40) are typically used as markers of stressors efficacy. We have observed that although behavioral and immunological alterations were only evident after exposing mice to 8 weeks of stress, suppression of body weight gain was observed as early as after 2 weeks of exposure and was maintained throughout the duration of CUS. These findings suggest that, as a read-out of the maladaptive response to stress, body weight gain has a lower threshold than other changes and is not a good marker of the stress-impact in behavior and/or immunity. Moreover, we did not observe a consistent reduction on thymus weight; although we cannot discard the possibility of being unable to detect small differences of thymus weight, specially given that mice were previously transcardially perfused with $0.9 \%$ saline. Nevertheless, the concomitant lack of differences in thymic cellularity favors our observation that, in C57BL/6 mice, our CUS protocol does not impact thymus weight significantly. This observation strengths the idea that C57BL/6 are more resistant to the effects of chronic stress than other mouse strains.

An overactive hypothalamic-pituitary-adrenal (HPA) axis is also a feature of a maladaptative response to chronic stress (41). In fact, resistance to chronic stress can be associated with an effective negative feedback system that is able to shut down the excessive production of glucocorticoids occurring in response to stress (42). We observed that the 8-week long CUS protocol was the only one that led to a persistent increase on circulating corticosterone levels and increased adrenals weight, features consistent with a hyperactive HPA axis. Of note, based on corticosterone levels at zenith we identified a reduced number of resistant animals ( 2 out of 10 in one of the experiments and 2 out of 10 in the replicated experiment), a proportion of stress-resistance very similar to what already have been described in other models of chronic stress (26). Accumulating evidence shows that glucocorticoids modulate the 
4 weeks

A

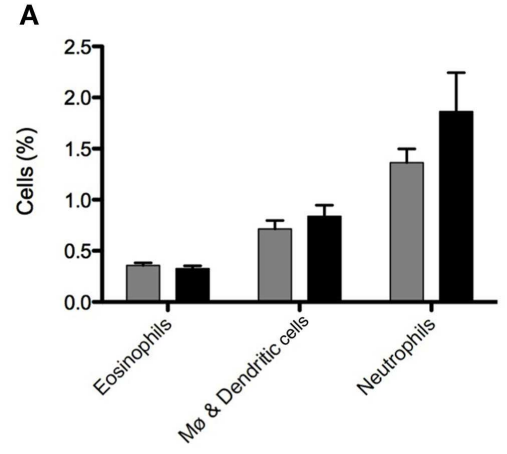

8 weeks

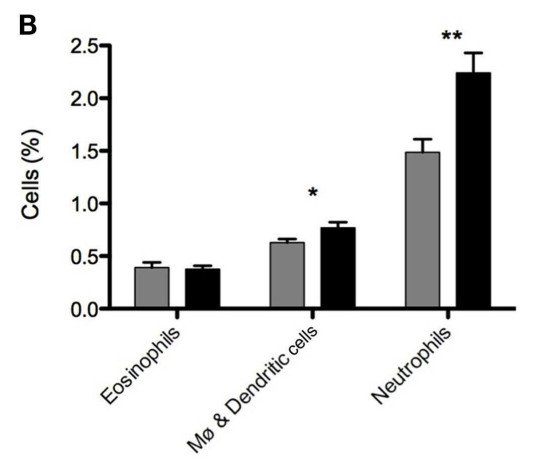

C

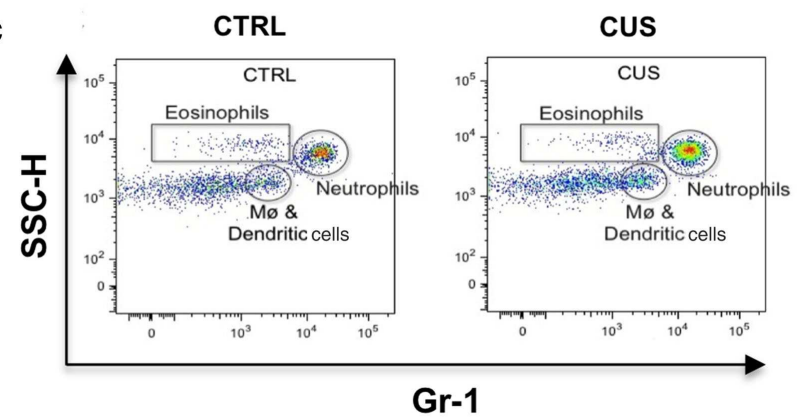

FIGURE 7 | Impact of 4 vs. 8 weeks of CUS on myeloid cellular populations in the spleen. Percentage of eosinophils, macrophages/dendritic cells, and neutrophils in spleen after exposure to
4 weeks (A) and 8 (B) weeks of CUS. Flow cytometry plot showing the gating strategy of myeloid splenocytes subsets (gates) (C). Each bar represents the mean \pm SEM from 8 animals per group. ${ }^{*} p<0.05,{ }^{* *} p<0.01$. behavioral effects of chronic stress $(43,44)$. In accordance, we observed that the 8-week long CUS protocol, the only that induced a hyperactive HPA axis, had a negative impact on emotional behavior. Specifically, we observed an enhanced anxious-like behavior, revealed by an increased time spent in the closed arms, and a decreased time in the open arms of the EPM. These animals also displayed behavioral despair, a symptom of depressive-like behavior, as they spent more time immobile in the FST. Of notice, this was further confirmed by performing the TST, another validated test for depressive-like behavior assessment.

Despite the emotional changes caused by 8 weeks of CUS exposure, cognitive functioning, namely spatial learning, seems to be intact, confirming data from other model of chronic stress (uCMS) (10). In fact, we observed that although stressed animals at the end of 4 days of MWM training were able to learn task at the same level as controls, there was a tendency for a slower learning progression on day 2 and 3. This type of learning pattern was previously shown using rats submitted to CUS (11), therefore emphasizing that the effects of CUS on spatial learning are more subtle than those on emotional behaviors. Contrary to the above mentioned effects, chronic stress triggers a decreased exploratory behavior of mice, both at 4 and 8 weeks of CUS, which might not be dependent on increased levels of corticosterone.

Although we cannot completely discard the possible confounder effect from performing two behavioral tests in the same day, we believe that data from the OF and TST was not significantly affected by acute stress caused by prior testing; indeed motor function (measured by OF) is not known as a target of acute stress, whereas data from TST were confirmed by the findings of the FST.

Glucocorticoids play a crucial role on thymopoiesis $(32,39)$, a process that occurs in the thymus in which immature precursor cells differentiate into mature $\mathrm{T}$ cells. In accordance, it was previously shown that rats exposed to chronic stress, with increased levels of circulating corticosterone levels, exhibit an increase in the percentage of DN thymocytes, while the percentage of SPCD4 was decreased (39). In our model, an increase in the percentage of DN thymocytes was observed. Still, contrary to the previously described (39), we did not observe any differences on the SPCD4 and SPCD8 populations of thymocytes, which may be due to the stress-resistance inherent to this particular strain of mice. $\mathrm{T}$ and B-lymphocytes in the spleen were not altered by exposure to chronic stress. However, we observed that exposure to 8 weeks of CUS (and not to 4 weeks) led to alterations in the cell composition of the spleen, characterized by an increased percentage of myeloid cells (macrophages/dendritic cells and neutrophils), in agreement with previous reports in both mice $(45,46)$ and humans $(46)$. Glucocorticoids were shown to inhibit neutrophils' apoptosis, which may explain the persistent presence of these cells with short life span (47) in the spleen of chronically stressed animals. Moreover, it was shown that stress, through norepinephrine signaling from sympathetic nerve fibers, increased the proliferation of hematopoietic progenitors in the bone marrow giving rise to an increase on disease-promoting monocytes and neutrophils output (46). Stress was also shown to 
increase monocyte recruitment to the brain by increased expression of cytokines and chemokines in specific brain regions. And more importantly, this monocyte recruitment to the brain was shown to be essential for the development of anxiety behavior induced by stress (45).

The absence of neuroendocrine, major behavioral and immunological alterations seen in the 4-week CUS exposed group could reflect the temporal dynamics of the stress-response rather than a failure to respond to stress. In fact, it should be noted that stress did impact the body weight gain and exploratory behavior on this group. This absence of major alterations resembles the Hans Selye's resistance phase of the so-called "syndrome of adaptation" (48) in which adaptative processes reinstall homeostasis during stress, including the normalization of glucocorticoid secretion. Therefore, the 4 -week CUS protocol may be preferable to studies that target this specific stage of the stress-response like for example those that want to show a negative impact of a particular treatment on the stress-response, since the 8-week CUS alterations may approach a "ceiling effect." In contrast, the alterations observed in the 8-week version of CUS are consistent with phase 3 of this syndrome, where the system is no longer able to cope with stressors and is exhausted, which renders this version a robust model of the maladaptative response to chronic stress.

The establishment of a robust mouse model of stress-related disorders on C57BL/6 background represents a valuable research tool endowing the study of different genetic contributions to chronic stress-responses, which may enhance current knowledge on the neurobiology and immunology of complex neuropsychiatric and other stress-related disorders.

\section{ACKNOWLEDGMENTS}

We acknowledge the Portuguese Science Foundation (FCT) for providing a fellowship to S. Monteiro (SFRH/BD/69311/2010) and S. Roque (SFRH/BPD/72710/2010).

\section{SUPPLEMENTARY MATERIAL}

The Supplementary Material for this article can be found online at http://www.frontiersin.org/Journal/10.3389/fpsyt.2015.00006/ abstract

\section{REFERENCES}

1. McEwen BS. Protection and damage from acute and chronic stress: allostasis and allostatic overload and relevance to the pathophysiology of psychiatric disorders. Ann N Y Acad Sci (2004) 1032:1-7. doi:10.1196/annals.1314.001

2. Glaser R, Kiecolt-Glaser JK. Stress-induced immune dysfunction: implications for health. Nat Rev Immunol (2005) 5:243-51. doi:10.1038/nri1571

3. Haroon E, Raison CL, Miller AH. Psychoneuroimmunology meets neuropsychopharmacology: translational implications of the impact of inflammation on behavior. Neuropsychopharmacology (2012) 37:137-62. doi:10.1038/npp. 2011.205

4. McEwen BS. Stressed or stressed out: what is the difference? J Psychiatry Neurosci (2005) 30:315-8.

5. Bondi CO, Rodriguez G, Gould GG, Frazer A, Morilak DA. Chronic unpredictable stress induces a cognitive deficit and anxiety-like behavior in rats that is prevented by chronic antidepressant drug treatment. Neuropsychopharmacology (2008) 33:320-31. doi:10.1038/sj.npp.1301410

6. Pego JM, Morgado P, Pinto LG, Cerqueira JJ, Almeida OF, Sousa N. Dissociation of the morphological correlates of stress-induced anxiety and fear. Eur J Neurosci (2008) 27:1503-16. doi:10.1111/j.1460-9568.2008.06112.x

7. McEwen BS, Eiland L, Hunter RG, Miller MM. Stress and anxiety: structural plasticity and epigenetic regulation as a consequence of stress. Neuropharmacology (2012) 62:3-12. doi:10.1016/j.neuropharm.2011.07.014
8. Ventura-Silva AP, Pego JM, Sousa JC, Marques AR, Rodrigues AJ, Marques F, et al. Stress shifts the response of the bed nucleus of the stria terminalis to an anxiogenic mode. Eur J Neurosci (2012) 36:3396-406. doi:10.1111/j.1460-9568. 2012.08262.x

9. Strekalova T, Spanagel R, Bartsch D, Henn FA, Gass P. Stress-induced anhedonia in mice is associated with deficits in forced swimming and exploration. Neuropsychopharmacology (2004) 29:2007-17. doi:10.1038/sj.npp. 1300532

10. Bessa JM, Mesquita AR, Oliveira M, Pego JM, Cerqueira JJ, Palha JA, et al. A trans-dimensional approach to the behavioral aspects of depression. Front Behav Neurosci (2009) 3:1. doi:10.3389/neuro.08.001.2009

11. Cerqueira JJ, Mailliet F, Almeida OF, Jay TM, Sousa N. The prefrontal cortex as a key target of the maladaptive response to stress. J Neurosci (2007) 27:2781-7. doi:10.1523/JNEUROSCI.4372-06.2007

12. Dias-Ferreira E, Sousa JC, Melo I, Morgado P, Mesquita AR, Cerqueira JJ, et al. Chronic stress causes frontostriatal reorganization and affects decision-making. Science (2009) 325:621-5. doi:10.1126/science.1171203

13. Conrad CD. A critical review of chronic stress effects on spatial learning and memory. Prog Neuropsychopharmacol Biol Psychiatry (2010) 34:742-55. doi:10.1016/j.pnpbp.2009.11.003

14. Morgado P, Silva M, Sousa N, Cerqueira JJ. Stress transiently affects Pavlovian-toinstrumental transfer. Front Neurosci (2012) 6:93. doi:10.3389/fnins.2012.00093

15. Dhabhar FS, McEwen BS. Acute stress enhances while chronic stress suppresses cell-mediated immunity in vivo: a potential role for leukocyte trafficking. Brain Behav Immun (1997) 11:286-306. doi:10.1006/brbi.1997.0508

16. Padgett DA, Glaser R. How stress influences the immune response. Trends Immunol (2003) 24:444-8. doi:10.1016/S1471-4906(03)00173-X

17. Dhabhar FS, Malarkey WB, Neri E, McEwen BS. Stress-induced redistribution of immune cells - from barracks to boulevards to battlefields: a tale of three hormones - Curt Richter Award winner. Psychoneuroendocrinology (2012) 37:1345-68. doi:10.1016/j.psyneuen.2012.05.008

18. Harpaz I, Abutbul S, Nemirovsky A, Gal R, Cohen H, Monsonego A. Chronic exposure to stress predisposes to higher autoimmune susceptibility in C57BL/6 mice: glucocorticoids as a double-edged sword. Eur J Immunol (2013) 43:758-69. doi:10.1002/eji.201242613

19. Franklin TB, Saab BJ, Mansuy IM. Neural mechanisms of stress resilience and vulnerability. Neuron (2012) 75:747-61. doi:10.1016/j.neuron.2012.08.016

20. Anisman H, Lacosta S, Kent P, McIntyre DC, Merali Z. Stressor-induced corticotropin-releasing hormone, bombesin, ACTH and corticosterone variations in strains of mice differentially responsive to stressors. Stress (1998) 2:209-20. doi:10.3109/10253899809167284

21. Anisman H, Hayley S, Kelly O, Borowski T, Merali Z. Psychogenic, neurogenic, and systemic stressor effects on plasma corticosterone and behavior: mouse strain-dependent outcomes. Behav Neurosci (2001) 115:443-54. doi:10.1037/0735-7044.115.2.443

22. Parfitt DB, Walton JR, Corriveau EA, Helmreich DL. Early life stress effects on adult stress-induced corticosterone secretion and anxiety-like behavior in the C57BL/6 mouse are not as robust as initially thought. Horm Behav (2007) 52:417-26. doi:10.1016/j.yhbeh.2007.06.002

23. Razzoli M, Carboni L, Andreoli M, Ballottari A, Arban R. Different susceptibility to social defeat stress of BalbC and C57BL6/J mice. Behav Brain Res (2011) 216:100-8. doi:10.1016/j.bbr.2010.07.014

24. Razzoli M, Carboni L, Andreoli M, Michielin F, Ballottari A, Arban R. Strainspecific outcomes of repeated social defeat and chronic fluoxetine treatment in the mouse. Pharmacol Biochem Behav (2011) 97:566-76. doi:10.1016/j.pbb. 2010.09.010

25. Savignac HM, Finger BC, Pizzo RC, O’Leary OF, Dinan TG, Cryan JF. Increased sensitivity to the effects of chronic social defeat stress in an innately anxious mouse strain. Neuroscience (2011) 192:524-36. doi:10.1016/j.neuroscience. 2011.04.054

26. Golden SA, Covington HE III, Berton O, Russo SJ. A standardized protocol for repeated social defeat stress in mice. Nat Protoc (2011) 6:1183-91. doi:10.1038/nprot.2011.361

27. Walf AA, Frye CA. The use of the elevated plus maze as an assay of anxiety-related behavior in rodents. Nat Protoc (2007) 2:322-8. doi:10.1038/nprot.2007.44

28. Can A, Dao DT, Arad M, Terrillion CE, Piantadosi SC, Gould TD. The mouse forced swim test. J Vis Exp (2012) 59:e3638. doi:10.3791/3638

29. Steru L, Chermat R, Thierry B, Simon P. The tail suspension test: a new method for screening antidepressants in mice. Psychopharmacology (Berl) (1985) 85:367-70. doi:10.1007/BF00428203 
30. Ottoni EB. EthoLog 2.2: a tool for the transcription and timing of behavior observation sessions. Behav Res Methods Instrum Comput (2000) 32:446-9. doi:10.3758/BF03200814

31. Rose S, Misharin A, Perlman H. A novel Ly6C/Ly6G-based strategy to analyze the mouse splenic myeloid compartment. Cytometry A (2012) 81:343-50. doi:10.1002/cyto.a.22012

32. Berki T, Palinkas L, Boldizsar F, Nemeth P. Glucocorticoid (GC) sensitivity and GC receptor expression differ in thymocyte subpopulations. Int Immunol (2002) 14:463-9. doi:10.1093/intimm/14.5.463

33. Nestler EJ, Hyman SE. Animal models of neuropsychiatric disorders. Nat Neurosci (2010) 13:1161-9. doi:10.1038/nn.2647

34. Yun J, Koike H, Ibi D, Toth E, Mizoguchi H, Nitta A, et al. Chronic restraint stress impairs neurogenesis and hippocampus-dependent fear memory in mice: possible involvement of a brain-specific transcription factor Npas4. J Neurochem (2010) 114:1840-51. doi:10.1111/j.1471-4159.2010.06893.x

35. Voorhees JL, Tarr AJ, Wohleb ES, Godbout JP, Mo X, Sheridan JF, et al. Prolonged restraint stress increases IL-6, reduces IL-10, and causes persistent depressivelike behavior that is reversed by recombinant IL-10. PLoS One (2013) 8:e58488. doi:10.1371/journal.pone.0058488

36. Bessa JM, Ferreira D, Melo I, Marques F, Cerqueira JJ, Palha JA, et al. The moodimproving actions of antidepressants do not depend on neurogenesis but are associated with neuronal remodeling. Mol Psychiatry (2009) 14(764-773):739. doi:10.1038/mp.2008.119

37. Dominguez-Gerpe L, Rey-Mendez M. Time-course of the murine lymphoid tissue involution during and following stressor exposure. Life Sci (1997) 61:1019-27. doi:10.1016/S0024-3205(97)00606-1

38. Ashwell JD, Lu FW, Vacchio MS. Glucocorticoids in T cell development and function. Annu Rev Immunol (2000) 18:309-45. doi:10.1146/annurev.immunol.18. 1.309

39. Zivkovic IP, Rakin AK, Petrovic-Djergovic DM, Kosec DJ, Micic MV. Exposure to forced swim stress alters morphofunctional characteristics of the rat thymus. J Neuroimmunol (2005) 160:77-86. doi:10.1016/j.jneuroim.2004.11.002

40. Ulrich-Lai YM, Figueiredo HF, Ostrander MM, Choi DC, Engeland WC, Herman JP. Chronic stress induces adrenal hyperplasia and hypertrophy in a subregion-specific manner. Am J Physiol Endocrinol Metab (2006) 291:E965-73. doi:10.1152/ajpendo.00070.2006

41. Sousa N, Almeida OF. Disconnection and reconnection: the morphological basis of (mal)adaptation to stress. Trends Neurosci (2012) 35:742-51. doi:10.1016/j.tins.2012.08.006
42. Taliaz D, Loya A, Gersner R, Haramati S, Chen A, Zangen A. Resilience to chronic stress is mediated by hippocampal brain-derived neurotrophic factor. J Neurosci (2011) 31:4475-83. doi:10.1523/JNEUROSCI.5725-10.2011

43. Cerqueira JJ, Pego JM, Taipa R, Bessa JM, Almeida OF, Sousa N. Morphological correlates of corticosteroid-induced changes in prefrontal cortex-dependent behaviors. J Neurosci (2005) 25:7792-800. doi:10.1523/JNEUROSCI.1598-05. 2005

44. Sousa N, Cerqueira JJ, Almeida OF. Corticosteroid receptors and neuroplasticity. Brain Res Rev (2008) 57:561-70. doi:10.1016/j.brainresrev.2007.06.007

45. Wohleb ES, Powell ND, Godbout JP, Sheridan JF. Stress-induced recruitment of bone marrow-derived monocytes to the brain promotes anxiety-like behavior. $J$ Neurosci (2013) 33:13820-33. doi:10.1523/JNEUROSCI.1671-13.2013

46. Heidt T, Sager HB, Courties G, Dutta P, Iwamoto Y, Zaltsman A, et al. Chronic variable stress activates hematopoietic stem cells. Nat Med (2014) 20(7):754-8. doi:10.1038/nm.3589

47. Saffar AS, Ashdown H, Gounni AS. The molecular mechanisms of glucocorticoids-mediated neutrophil survival. Curr Drug Targets (2011) 12:556-62. doi:10.2174/138945011794751555

48. Selye H. A syndrome produced by diverse nocuous agents. Nature (1936) 138:32-32. doi:10.1038/138032a0

Conflict of Interest Statement: The authors declare that the research was conducted in the absence of any commercial or financial relationships that could be construed as a potential conflict of interest.

Received: 13 September 2014; accepted: 13 January 2015; published online: 02 February 2015.

Citation: Monteiro S, Roque S, de Sá-Calçada D, Sousa N, Correia-Neves M and Cerqueira JJ (2015) An efficient chronic unpredictable stress protocol to induce stressrelated responses in C57BL/6 mice. Front. Psychiatry 6:6. doi: 10.3389/fpsyt.2015.00006 This article was submitted to Affective Disorders and Psychosomatic Research, a section of the journal Frontiers in Psychiatry.

Copyright (c) 2015 Monteiro, Roque, de Sá-Calçada, Sousa, Correia-Neves and Cerqueira. This is an open-access article distributed under the terms of the Creative Commons Attribution License (CC BY). The use, distribution or reproduction in other forums is permitted, provided the original author(s) or licensor are credited and that the original publication in this journal is cited, in accordance with accepted academic practice. No use, distribution or reproduction is permitted which does not comply with these terms. 